[0212-7199 (2008) 25: 6; pp 256-261] ANALES DE MEDICINA INTERNA Copyright (C) 2008 ARAN EDICIONES, S.L.

AN. MED. INTERNA (Madrid) Vol. 25, N. ${ }^{\circ} 6$, pp. 256-261, 2008

\section{Factores epidemiológicos asociados a la hospitalización por descompensación de la insuficiencia cardiaca}

\author{
A. HERMIDA AMEIJEIRAS, M. PAZO NÚÑEZ, R. DE LA FUENTE CID, \\ F. L. LADO LADO, L. HERNÁNDEZ FERNÁNDEZ, I. RODRÍGUEZ LÓPEZ \\ (GRUPO SANTICOR)
}

Servicio de Medicina Interna. Departamento de Medicina. Complejo Hospitalario Universitario. Santiago de Compostela. A Coruña
EPIDEMIOLOGICAL EVENTS RELATED TO DECOMPENSATED HEART FAILURE

\begin{abstract}
RESUMEN
Objetivo: El presente estudio pretende conocer el perfil del paciente que ingresa por descompensación de insuficiencia cardíaca en un hospital de tercer nivel asistencial.

Métodos: Se diseña un estudio observacional y retrospectivo en el que se registra de forma aleatoria los ingresos por este proceso en nuestro centro durante el año 2005.

Resultados: El tamaño muestral es de 209 pacientes (media de edad: $78,6 \pm 9,1 ; 52,4 \%$ varones), con un índice de comorbilidades del 87,55\%. Casi un tercio de los pacientes no disponen de valoración de la función sistólica y entre los restantes, la gran mayoría $(72,4 \%)$ presentan función sistólica conservada. La gran mayoría de las descompensaciones surgen en el seno de infecciones respiratorias. La cardiopatía isquémico-hipertensiva es el origen más frecuente de la cardiopatía con disfunción sistólica. Se registró una estancia hospitalaria media de 12,9 días con un índice de mortalidad del 9,56\% resultando sus principales factores de riesgo estadíos funcionales avanzados en las escalas de la NYHA o de la Cruz Roja así como la presencia de demencia o ictus.

Conclusiones: El presente estudio muestra un perfil del paciente hospitalizado por descompensación de insuficiencia cardíaca que difiere notablemente de aquel incluído en los grandes ensayos clínicos, lo que sin duda dificulta la aplicación de estrategias terapéuticas que han demostrado ser útiles en aquellos casos.
\end{abstract}

PALABRAS CLAVE: Insuficiencia cardíaca. Mortalidad. Epidemiología. Hospitalización.

\begin{abstract}
Objective: The present study aimed to evaluate the profile of patients with decompensated heart failure hospitalized in a tertiary hospital.

Methods: It was designed an observational and retrospective study where data from clinical records of patients suffering from heart failure along 2005 were registered randomly.

Results: 209 patients were collected (average age: $78.6 \pm 9.1$; male: $52.4 \%$ ) with a comorbidity rate of $87.55 \%$. Almost one third of them have not stimation of systolic function and among the others $72.4 \%$ have it preserved. Most of decompensated were due to respiratory infections. Ischemic-hipertensive cardiopathy was the most frequent aetiology of systolic disfunction. Average stay was 12.9 days with a mortality rate of 9.56\%. Its main risk factors were advanced stages in NYHA od Red Cross scales, as so as dementia or ictus.

Conclusions: The present study shows a patient hospitalized for decompensated heart failure roughly different from that one reported at clinical trials. It makes difficult to apply therapeutical interventions, previously well documented to be useful.
\end{abstract}

KEY WORDS: Heart failure. Mortality. Epidemiology. Hospitalization.

Hermida Ameijeiras A, Pazo Núñez M, de la Fuente Cid R, Lado Lado FL, Hernández Fernández L, Rodríguez López I (Grupo SANTICOR). Factores epidemiológicos asociados a la hospitalización por descompensación de la insuficiencia cardíaca. An Med Interna (Madrid) 2008; 25: 256-261.

\section{INTRODUCCIÓN}

La insuficiencia cardíaca (IC) continúa siendo en nuestros días, un creciente problema de salud pública que se acompaña de importante morbilidad y mortalidad. En EE.UU. se producen anualmente alrededor de 1.099.000 ingresos hospitalarios por descompensación de la IC con un coste aproximado de 32,2 billones de dólares (1). Mientras, en nuestro país, la IC afecta al $10 \%$ de la población mayor de 70 años y se producen anualmente alrededor de 80.000 ingresos hospitalarios por descompensación de la misma (2), constituyendo la tercera causa de muerte cardiovascular por detrás de la cardiopatía isquémica y el accidente cerebrovascular.

Trabajo aceptado: 08 de febrero de 2008

Correspondencia: Álvaro Hermida Ameijeiras. Servicio de Medicina Interna. Hospital Clínico. Avda. Choupana, s/n. 15706 Santiago de Compostela (A Coruña). Fax: 981-950501.e-mail: ameijeiras@sergas.es 
Son factores asociados a la morbimortalidad así como a la calidad de vida de los pacientes con IC el sexo, la edad, la fracción de eyección del ventrículo izquierdo (FEVI), el estado nutricional o los trastornos depresivos entre otros $(3,4)$. Algunas series han llegado a constatar una tasa de reingreso por descompensación de la IC de alrededor del 30\% en los tres meses siguientes al alta hospitalaria (5).

Así y todo, la prevalencia de la IC sigue aumentando debido en parte al descenso de la mortalidad en los últimos años $(6,7)$ y constituye la primera causa de hospitalización en mayores de 65 años en los Servicios de Medicina Interna (2). Por este motivo resulta de especial interés, identificar los factores que influyen en la hospitalización por este síndrome, tanto en aquellos pacientes con FEVI disminuída donde existe una amplia evidencia científica y la estrategia terapéutica está bien definida (8) como aquellos con FEVI conservada donde prevalece la controversia en relación con múltiples variables incluyendo prevalencia, aspectos clínicos o pronósticos (9).

El objetivo del presente estudio fue evidenciar perfiles epidemiológicos que condicionen el riesgo de hospitalización por descompensación de la IC, una vez que las terapias basadas en la evidencia han conseguido incrementar la supervivencia en estos pacientes.

\section{MATERIAL Y MÉTODOS}

Este estudio observacional está basado en el análisis retrospectivo y aleatorizado de los episodios de hospitalización por descompensación de IC en el Servicio de Medicina Interna del Complejo Hospitalario Universitario de Santiago de Compostela desde el 01 de enero de 2005 al 31 de diciembre del mismo año. Es este un centro sanitario de tercer nivel cuya área asistencial comprende alrededor de 418.965 habitantes. De forma confidencial y a través de los registros de historias clínicas e informes médicos al alta, se recogieron datos demográficos de los pacientes así como sus antecedentes clínicos de interés. De igual modo se obtuvieron los factores desencadenantes que motivaron la descompensación de la IC así como la etiología de la cardiopatía y el estadío evolutivo de la misma. Finalmente, se registraron variables pronósticas como la estancia hospitalaria y la mortalidad total. No se excluyeron aquellos pacientes con más de un episodio de hospitalización incluído en el mismo período del estudio.

Se consideró IC con función sistólica deprimida aquellos con FEVI $<45 \%$, estimado mediante técnica ecocardiográfica tal y como establece la Sociedad Europea de Cardiología (10).

Para el análisis estadístico se utilizó el programa SPSS (versión 11.0), empleando las frecuencias absolutas y porcentajes para la medición de variables nominales y el test t-Student para muestras independientes en la comparación de medias entre variables continuas así como el test $\chi^{2}$ y el test exacto de Fischer o de Mc Nemar (con la corrección de Yates donde corresponda) para variables categóricas y el cálculo del riesgo relativo. La significación estadística se estableció para un valor de $\mathrm{p}<0,05$ y un intervalo de confianza del $95 \%$.

\section{RESULTADOS}

El número total de pacientes objeto de estudio en el período citado fue de 209 (varones: 52,4\%) con una media de edad de 78,6 años (DS: \pm 9,1; mediana: 80; min: 34; max: 94).

Un $87,55 \%$ de los pacientes presentaban comorbilidades asociadas de entre las cuales destacaba la hipertensión arterial, presente en el 61,2\% del total. Otros antecedentes clínicos de interés fueron la diabetes mellitus $(30,4 \%)$, la enfermedad pulmonar obstructiva crónica $(30,4 \%)$; la insuficiencia renal crónica (10\%), la patología del sistema nervioso central (demencia, ictus) $(15,8 \%)$ y la hepatopatía crónica $(2,4 \%)$. Entre otros factores de riesgo cardiovascular además de la hipertensión destacaron la obesidad con un 34,9\%, el tabaquismo $(21,5 \%)$ y la dislipemia $(19,1 \%)$. En la tabla I se representan estos y otros datos demográficos de los pacientes objeto de estudio.

\section{TABLA I}

DATOS DEMOGRÁFICOS Y CONDICIONES CLÍNICAS DE INTERÉS RELATIVOS A LOS PACIENTES RECOGIDOS EN EL ESTUDIO

\begin{tabular}{ll}
\hline & $\begin{array}{l}N(\%) \text { (para variables numéricas: } \\
\text { media } \pm \text { desviación típica) }\end{array}$ \\
\hline Sexo (varón) & $109(52,4)$ \\
\hline Edad & $78,62 \pm 9,1$ \\
\hline Apoyo social & \\
Residencia asistidos & $13(6,3)$ \\
Familia & $166(80,6)$ \\
Cuidador & $19(9,2)$ \\
Solo & $8(3,9)$ \\
\hline Nivel de estudios & \\
Analfabeto & $5(2,4)$ \\
Alfabetización & $143(69,4)$ \\
Estudios primarios & $56(27,2)$ \\
Estudios superiores & $2(1)$ \\
\hline Estado civil & \\
Soltero & $13(6,5)$ \\
Casado & $92(45,8)$ \\
Viudo & $96(47,8)$ \\
\hline
\end{tabular}

Grado de incapacidad mental en la

Escala de la Cruz Roja

।

$\|$

III

IV

$\mathrm{V}$

$16(7,9)$

$70(34,5)$

$51(25,1)$

$45(22,2)$

$17(8,4)$

$4(2)$

Grado de incapacidad física en la

Escala de la Cruz Roja

$0 \quad 4$ (2)

I $\quad 44(21,6)$

II $\quad 81(39,7)$

III $\quad 44(21,6)$

IV $\quad 31(15,2)$

Clase funcional de la NYHA

I $\quad 23(11,5)$

|| $102(51)$

III $\quad 69(34,5)$

IV 6(3) 
TABLA I (CONTINUACIÓN)

DATOS DEMOGRÁFICOS Y CONDICIONES CLÍNICAS DE INTERÉS RELATIVOS A LOS PACIENTES RECOGIDOS EN EL ESTUDIO

\begin{tabular}{ll}
\hline & $N(\%)$ (para variables numéricas: \\
& media \pm desviación típica) \\
\hline Antecedentes clínicos & \\
Hipertensión arterial & $128(61,2)$ \\
Diabetes mellitus & $63(30,4)$ \\
EPOC & $63(30,4)$ \\
Insuficiencia renal crónica & $21(10)$ \\
Hepatopatía crónica & $5(2,4)$ \\
Patología del SNC (ictus,demencia) & $33(15,8)$ \\
Tabaquismo & $45(21,5)$ \\
Dislipemia & $40(19,1)$ \\
Obesidad (IMC>30) & $73(34,9)$ \\
\hline Número de fármacos al ingreso & $6,26+1-2,78$ \\
\hline Estancia hospitalaria & $12,9+/-8,71$ \\
\hline Etiología & \\
Hipertensiva & $127(60,8)$ \\
Isquémica & $52(24,9)$ \\
Miocardiopatía dilatada & $5(2,4)$ \\
Valvulopatía & $73(34,9)$ \\
Secundaria a tóxicos & $1(0,5)$ \\
Cor pulmonale & $37(17,7)$ \\
Otras & $4(1,9)$ \\
Desconocida & $22(10,5)$ \\
\hline Factor precipitante de la descompensación & \\
Infección respiratoria & $115(55)$ \\
Fármacos & $1(0,5)$ \\
Crisis hipertensiva & $6(2,9)$ \\
Incumplimiento terapéutico & $8(3,8)$ \\
Anemia & $38(18,2)$ \\
Arritmia no controlada & $25(12)$ \\
Cardiopatía isquémica & $10(4,8)$ \\
Estrés emocional & $3(1,4)$ \\
Otros & $19(9,1)$ \\
Desconocido & $29(13,9)$ \\
\hline Fracción de eyección del VI & \\
- 45\% & \\
- 30-45\% & \\
- <30\% & \\
\hline & \\
& \\
\hline
\end{tabular}

Del total de pacientes, 60 de ellos $(30,6 \%)$ no presentaban estudio ecocardiográfico ni angiográfico reciente, por lo que fue imposible la estimación de la FEVI y la subsiguiente diferenciación entre cardiopatías con función sistólica deprimida o conservada. Del resto de pacientes, el 72,4\% presentaban FEVI $>45 \%$. No se registraron diferencias estadísticamente significativas en relación con aspectos demográficos o antecedentes clínicos entre ambos grupos.

El análisis de los factores precipitantes que motivaron la descompensación de la IC y posterior hospitalización tampoco objetivó diferencias significativas entre ambos tipos de car- diopatía. La infección respiratoria fue el principal causante en ambos grupos con un $60 \%$ en pacientes con FEVI disminuida (47,6\% con FEVI normal) seguido del síndrome anémico con un 20 y un $19 \%$ respectivamente, las arritmias no controladas representaron un 10 y un $9,5 \%$ en cada caso y la cardiopatía isquémica un 10 y un 5,7\%. La crisis hipertensiva se identificó como responsable de un $2,5 \%$ en los pacientes con disfunción sistólica (frente al 2,9\%) y el incumplimiento terapéutico en un $10 \%$ (frente al 2,9\%). Finalmente se objetivó un $1,9 \%$ de pacientes con función sistólica conservada cuyo factor precipitante para la descompensación de su cardiopatía fue el estrés emocional.

El análisis comparativo del origen de la cardiopatía entre aquellos con función sistólica deprimida y conservada revela que existen diferencias estadísticamente significativas con respecto a la cardiopatía isquémica con un 52,5 y un $25,7 \%$ respectivamente (RR: 1,43; IC 95\%: 1,09-1,26) así como con la miocardiopatía dilatada con un 10 y un $1 \%$ respectivamente (RR: 3,71; IC 95\%: 0,6-21,4). La distribución porcentual de estas y otras etiologías de la cardiopatía de base se representan en la figura 1.

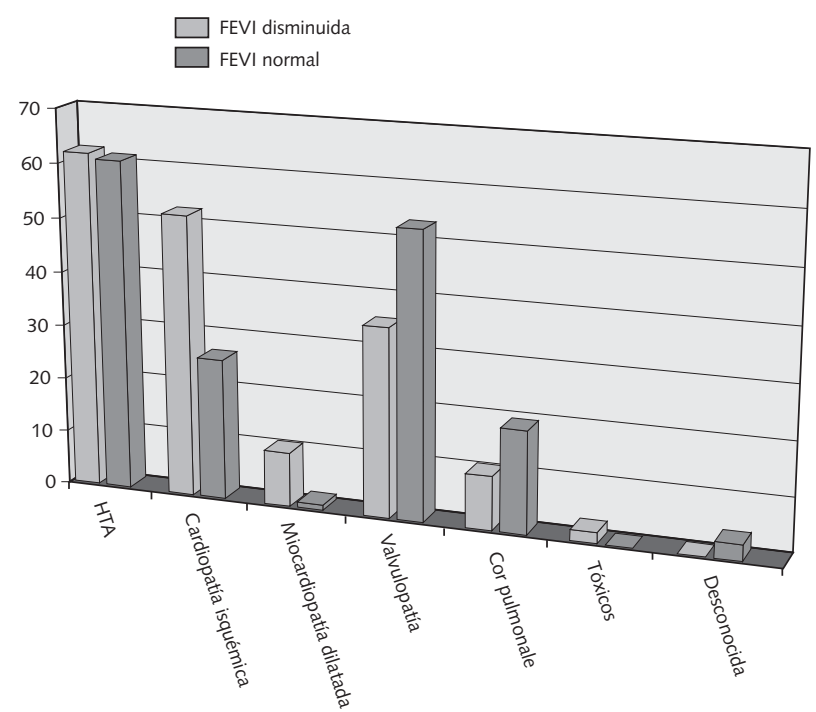

Fig. 1. Representación gráfica de la distribución porcentual de etiología de la cardiopatía según FEVI. Gupo SANTICOR.

De los 209 pacientes del estudio, 20 resultaron éxitus $(9,56 \%)$ con una media de edad de 78,6 años (DS: $\pm 9,1)$ de los cuales, un $52,4 \%$ fueron varones. No se objetivaron diferencias significativas en la mortalidad en relación con la edad, sexo, apoyo social, nivel de estudios, estado civil, seguimiento previo al ingreso, antecedentes clínicos, precipitantes de la descompensación, etiología de la cardiopatía o FEVI (Tabla II).

Existe un incremento de la mortalidad con clases funcionales de la NYHA III-IV, aunque esta asociación no cumple criterios de significación estadística. De igual modo, un grado de incapacidad física en la escala de la Cruz Roja $(E C R) \geq 3$ se correlaciona con el doble de probabilidades de fallecimiento (RR: 2,88; IC 95\%: 1,12-7,41). Se demostró correlación con la estancia media hospitalaria (éxitus: 19,46 días; no éxi- 
TABLA II

TABLA DE CONTINGENCIA DE MORTALIDAD EN RELACIÓN A DISTINTAS VARIABLES

\begin{tabular}{|c|c|c|c|c|}
\hline & $\begin{array}{c}\text { Frecuencia } \\
\text { en exitus }\end{array}$ & $\begin{array}{c}\text { Frecuencia en } \\
\text { no exitus }\end{array}$ & Significación & $\begin{array}{c}\text { Riesgo relativo/ } \\
\text { Intervalo } \\
\text { confianza } 95 \%\end{array}$ \\
\hline SEXO & & & $p=0,49$ & \\
\hline Varón & $11,9 \%$ & $88,1 \%$ & & \\
\hline Mujer & $8,1 \%$ & $91,9 \%$ & & \\
\hline APOYO SOCIAL & & & $p=0,28$ & \\
\hline Residencia & $14,3 \%$ & $5,4 \%$ & & \\
\hline Familia & $81 \%$ & $80,5 \%$ & & \\
\hline Cuidador & $4,8 \%$ & $9,7 \%$ & & \\
\hline Solo & $0 \%$ & $4,3 \%$ & & \\
\hline NIVEL ESTUDIOS & & & $p=0,24$ & \\
\hline Analfabeto & $0 \%$ & $2,7 \%$ & & \\
\hline Alfabetización & $71,4 \%$ & $69,2 \%$ & & \\
\hline Primarios & $23,8 \%$ & $27,6 \%$ & & \\
\hline Superiores & $4,8 \%$ & $0,5 \%$ & & \\
\hline ESTADO CIVIL & & & $p=0,79$ & \\
\hline Soltero & $10 \%$ & $6,1 \%$ & & \\
\hline Casado & $45 \%$ & $45,9 \%$ & & \\
\hline Viudo & $45 \%$ & $48,1 \%$ & & \\
\hline SEGUIMIENTO PREVIO & & & $p=0,87$ & \\
\hline Ninguno & $15 \%$ & $11,5 \%$ & & \\
\hline At. primaria & $55 \%$ & $47,3 \%$ & & \\
\hline Internista & $20 \%$ & $28 \%$ & & \\
\hline Cardiólogo & $10 \%$ & $13,2 \%$ & & \\
\hline
\end{tabular}

\section{ANTECEDENTES CLÍNICOS}

\begin{tabular}{|c|c|c|c|c|}
\hline HTA & $66,7 \%$ & $60,6 \%$ & $p=0,64$ & \\
\hline Diabetes mellitus & $23,8 \%$ & $31,2 \%$ & $p=0,62$ & \\
\hline EPOC & $38,1 \%$ & $29,3 \%$ & $p=0,25$ & \\
\hline IRC & $14,3 \%$ & $9,3 \%$ & $p=0,45$ & \\
\hline Hepatopatía & $0 \%$ & $2,7 \%$ & $p=1$ & \\
\hline Patología Previa SNC & $33,3 \%$ & $13,8 \%$ & $p=0,029$ & $3,11 / 1,14-8,44$ \\
\hline Tabaquismo & $23,8 \%$ & $21,3 \%$ & $p=0,78$ & \\
\hline Dislipemia & $33,3 \%$ & $17,6 \%$ & $p=0,13$ & \\
\hline Etilismo moderado & $0 \%$ & $2,7 \%$ & $p=1$ & \\
\hline Obesidad & $19 \%$ & $36,7 \%$ & $p=0,14$ & \\
\hline NYHA & & & $p=0,074$ & \\
\hline Clase I & $0 \%$ & $12,7 \%$ & & \\
\hline Clase II & $36,8 \%$ & $52,5 \%$ & & \\
\hline Clase III & $57,9 \%$ & $32 \%$ & & \\
\hline Clase IV & $5,3 \%$ & $2,8 \%$ & & \\
\hline
\end{tabular}

FACTOR PRECIPITANTE

\begin{tabular}{lccc} 
Infección & $61,9 \%$ & $54,3 \%$ & $p=0,6$ \\
Fármacos & $0 \%$ & $0,5 \%$ & $p=1$ \\
Inestabilidad PA & $0 \%$ & $3,2 \%$ & $p=1$ \\
Incumplimiento tto. & $9,5 \%$ & $3,2 \%$ & $p=0,18$ \\
Anemia & $23,8 \%$ & $17,6 \%$ & $p=0,54$ \\
Arritmia & $9,5 \%$ & $12,2 \%$ & $p=1$ \\
Cardiopatía Isquémica & $9,5 \%$ & $4,3 \%$ & $p=0,26$ \\
Estrés emocional & $0 \%$ & $1,6 \%$ & $p=1$ \\
\hline
\end{tabular}

\begin{tabular}{|c|c|c|c|c|}
\hline \multicolumn{5}{|l|}{ ETIOLOGIIA CARDIOPATÍA } \\
\hline Hipertensiva & $57,1 \%$ & $61,2 \%$ & $p=0,81$ & \\
\hline Isquémica & $28,6 \%$ & $24,5 \%$ & $p=0,79$ & \\
\hline Dilatada & $0 \%$ & $2,7 \%$ & $p=1$ & \\
\hline Valvulopatía & $28,6 \%$ & $35,6 \%$ & $p=0,63$ & \\
\hline Cor pulmonale & $23,8 \%$ & $17 \%$ & $p=0,54$ & \\
\hline FRACCIÓN EYECCIÓN VI & & $p=0,29$ & & \\
\hline$->45 \%$ & $75 \%$ & $72,2 \%$ & & \\
\hline$-45-30 \%$ & $25 \%$ & $14,3 \%$ & & \\
\hline$-<30 \%$ & $0 \%$ & $13,5 \%$ & & \\
\hline ESCALA INCAPACIDAD & & & & \\
\hline MENTAL CRUZ ROJA $\geq 3$ & $30 \%$ & $8,2 \%$ & $p=0,009$ & $4,8 / 1,61-14,31$ \\
\hline ESCALA INCAPACIDAD & & & & \\
\hline FÍSICA CRUZ ROJA $\geq 3$ & $60 \%$ & $34,2 \%$ & $p=0,029$ & $2,88 / 1,12-7,41$ \\
\hline
\end{tabular}

tus: 12,3 días) y también con grados de incapacidad mental en la ECR $\geq 3$ o con patología previa del SNC (RR: 3,11 ; IC 95\%: $1,14-8,44)$.

\section{DISCUSIÓN}

La hospitalización por descompensación de IC continúa siendo un proceso frecuente tanto en pacientes con disfunción sistólica como en aquellos con FEVI conservada (11). Los resultados derivados del presente estudio reflejan un perfil demográfico similar al obtenido por otras series publicadas dentro y fuera del estado español $(6,12-13)$. Si bien es cierto que nuestros pacientes tienden a ser sensiblemente mayores que en el caso de los EE.UU. (con una media de edad de 72,4 años en la serie del registro ADHERE) (6), lo que le confiere mayor complejidad al proceso y sin duda contribuye al mayor índice de mortalidad y estancia media hospitalaria registrado en series nacionales.

Continúa siendo especialmente reseñable el elevado índice de comorbilidades presente en pacientes con IC, alcanzando en nuestra serie hasta un $87 \%$ de los mismos, con una distribución similar a otras publicadas con anterioridad (14). Cabe recordar que la presencia de comorbilidades incide directamente sobre la complejidad del proceso afectando al pronóstico, tratamiento, seguimiento y calidad de vida de los pacientes con IC, además de un incremento del gasto sanitario (15). El nivel de comorbilidad es mayor en los pacientes manejados por el médico internista (16) y ello limita la aplicación de las recomendaciones derivadas de grandes ensayos clínicos y adoptadas por las sociedades científicas ya que pacientes de edad tan avanzada y con alta prevalencia de enfermedades asociadas no son habitualmente incluídos en dichos ensayos.

De modo similar, el empleo de técnicas diagnósticas complementarias como la ecocardiografía, durante la hospitalización por descompensación de IC, es menor entre médicos internistas en comparación con especialistas en cardiología, lo que se ha puesto en relación con la dificultad o facilidad de acceso a dichas pruebas, además de las particularidades de los pacientes hospitalizados en cada Servicio o Unidad ya que como se ha mencionado, la media de edad e índice de comorbilidades es mayor entre los primeros y ello puede condicio- 
nar el grado de aproximación diagnóstica con mayor o menor agresividad en cada caso $(16,17)$. En nuestra serie, un tercio de los pacientes carecían de estimación de la FEVI, un porcentaje inferior al presentado en otras series $(14,16)$ y que confirma una tendencia ya evidenciada por otros autores de incremento en el empleo de pruebas complementarias para mejor filiación de la cardiopatía de base (6). Ello puede estar indicando una mayor concienciación y un intento por adecuarse a recomendaciones basadas en la evidencia que establecen claramente directrices en un sentido $u$ otro asociadas a un incremento de la supervivencia. Sin embargo, los autores consideran que estamos todavía lejos de alcanzar el estándar de calidad y debemos realizar un esfuerzo complementario en este sentido. Sin duda, las unidades clínicas monográficas y multidisciplinares para el diagnóstico, tratamiento y seguimiento de la IC son herramientas indispensables para la consecución de dicho objetivo.

La identificación y actuación precoz sobre los factores precipitantes de un episodio de descompensación de IC debe continuar siendo una prioridad. En nuestra serie, la infección respiratoria representa el principal desencadenante de la descompensación; independientemente del grado de función sistólica.

El análisis del origen de la cardiopatía en nuestra serie evidencia la multifactorialidad etiológica en el $45 \%$ de los pacientes, siendo la hipertensión arterial sin duda la más prevalente, presente en el $60,8 \%$ de los casos con una distribución equiparable según FEVI. El resto de factores etiológicos sí presentan variación según la función sistólica y en el caso de la cardiopatía isquémica o la miocardiopatía dilatada, con significación estadística. Estas diferencias previamente constatadas por otros autores $(12,14,18)$, refuerzan la idea de que el abordaje terapéutico debe ser diferente atendiendo al origen de la IC y se requieren nuevas evidencias para aquellos con función sistólica conservada, que representan en nuestra serie el $72,4 \%$, un porcentaje superior al documentado en otras publicaciones $(13,14)$ y que puede estar reflejando una incidencia creciente para este subgrupo de cardiópatas ya evidenciada con anterioridad (6). Tal vez el mayor control de los factores de riesgo que condicionan la aparición de IC con

\section{Bibliografía}

1. American Heart Association. Herat Disease and Stroke Statistics_2007 Update. http://www.americanheart.org.

2. Rodríguez Artalejo F, Banegas Banegas JR, Guallar Castillón P. Epidemiología de la insuficiencia cardíaca. Rev Esp Cardiol 2004; 57: 163170.

3. Alla F, Al-Hindi AY, Lee CR, Schwartz TA, Herbert Patterson J, Adams KF, Jr. Relation of sex to morbidity and mortality in patients with Heart failure and reduced or preserved left ventricular ejection fraction. Am Heart J 2007; 153: 1074-80.

4. Etxeberría-Lekuona D, Sánchez-Álvarez J, Alonso Gutiérrez A, Acha Arrieta V, Campos Rivas R, Jarne Betrán V. Study of quality of life of patients with Heart failure in an Internal Medicine Department. An Med Interna (Madrid) 2007; 24: 57-60.

5. Krumholz HM, Parent EM, Tu N, Vaccarino V, Wang Y, Radford MJ, Hennen J. Readmission after hospitalization for congestive heart failureamong Medicare beneficiaries. Arch Intern Med 1997; 157: 99-104.

6. Fonarow GC, Heywood JT, Heidenreich PA, Lopatin M, Yancy CW, for the ADHERE Scientific Advisory Committee and Investigators. Temporal trends in clinical characteristics, treatments, and outcomes disfunción sistólica y en concreto la cardiopatía isquémica, cuya incidencia poblacional está prácticamente estabilizada desde 1990 tal y como se refleja en el registro REGICOR (19), sea responsable de esta tendencia.

Finalmente los resultados del presente estudio reflejan una estancia media por proceso de descompensación de IC de 12,9 días (DS: $\pm 8,7$ ) sin objetivarse diferencias significativas en base a la FEVI. El índice de mortalidad se sitúa por encima del 9\% y está íntimamente relacionado con el grado de limitación funcional en el paciente medido por las escalas de la NYHA o de incapacidad física y mental de la ECR así como por la presencia de ictus o demencia. Estos hallazgos concuerdan en gran medida con otras series nacionales y europeas $(13,16)$. No así sin embargo con los pacientes del registro ADHERE en los EE.UU. (6), con unos índices de mortalidad y estancia media hospitalaria claramente inferiores y que tal y como se comentó con anterioridad, pudiera estar en relación con las diferencias demográficas e índice de comorbilidades ya constatadas.

Los resultados derivados del presente estudio refleja la realidad de nuestros pacientes hospitalizados por descompensación de IC. Una realidad que difiere en parte de aquella otra mostrada por los grandes ensayos clínicos, cuyos pacientes suelen ser más jóvenes, en clases funcionales menos avanzadas y con un menor índice de comorbilidades. Ello contribuiría a explicar la dificultad que nos encontramos en nuestra práctica clínica habitual para implementar las estrategias terapéuticas que han demostrado ser de mayor utilidad en dichos ensayos.

\section{AGRADECIMIENTOS}

Al conjunto de integrantes del Grupo SANTICOR; Dr. Lado Lado F.L.; Dr. Rodríguez López, I. Prof. Dr. Lorenzo Zúñiga, V.; Dr. de la Fuente Cid, R.; Dra. Domínguez Santalla, M.J.; Dr. Rodríguez Moreno, C.; Dr. Hermida Ameijeiras, A.; Dra. Pazo Núñez, M.; Dra. Hernández Fernández, L.; Dra. Mallo González, N.; Dra. Barros Alcalde, P.; Dra. Roca Pardiñas, L.; Dra. Fernández Conde, S.; Dra. Freire Ramos, A.; Dr. Fernández Somoza, J.; Dra. Valcárcel Rodriguez, C. for heart failure hospitalizations, 2002 to 2004: findings from Acute Decompensated Heart Failure National Registry (ADHERE). Am Heart J 2007; 153: 1021-8.

7. Cleland J, Khand A, Clark A. The heart failure epidemic: exactly how big is it? Eur Heart J 2001; 22: 623-26.

8. Hunt SA, Abraham WT, Chin MH, et al. ACC/AHA 2005 guideline update for the diagnosis and management of chronic heart failure in the adult: a report of the American College of Cardiology/American Heart Association Task Force on Practice Guidelines (Writing Committee to Update the 2001 Guidelines for the Evaluation and Management of Heart Failure). J Am Coll Cardiol 2005; 46: 1116-43.

9. Burkhoff D, Maurer MS, Packer M. Heart failure with a normal ejection fraction: is it really a disorder of diastolic function? Circulation 2003; 107: 656-658.

10. How to diagnose diastolic heart failure. European Study group on Diastolic Heart failure. Eur Heart J 1998; 19: 990-1003.

11. Hogg K, Swedberg K, McMurray J. Heart failure with preserved left ventricular systolic function; epidemiology, clinical characteristics and prognosis. J Am Coll Cardiol 2004; 43: 317-27. 
12. Torres-Gárate R, Álvarez-Rodríguez E, Segoviano Mateo R, GutiérrezLarraínzar A, Lozano Tonkin C, Calvo Manuel E. Clinical and therapeutic characteristics in patients with heart failure cared in a tertiary hospital. Rev Clin Esp 2005; 205: 322-5.

13. Fernandez Galante I, Gonzalez Sarmiento E. Clinical and therapeutic characteristics in patients with Heart failure cared for in an internal medicine department. An Med Interna (Madrid) 2006; 23: 459-64.

14. Grupo de Trabajo de Insuficiencia Cardíaca de la Sociedad Española de Medicina Interna. La insuficiencia cardíaca en los Servicios de Medicina Interna (estudio SEMI-IC). Med Clin 2002; 118: 605-10.

15. Poses RM, McClish DK, Smith WR. Results of report cards for patients with congestive heart failure depend on the method used to adjust for severity. Ann Intern Med 2000; 133: 10-20.

16. Belloti P, Badano LP, Acquarone N, Griffo R, Lo Pinto G, Maggioni
AP, et al. Specialty-related differences in the epidemiology, clinical profile, management and outcome of patients hospitalizad for heart failure. The OSCUR study. Eur Heart J 2001; 22: 596-604.

17. Reis SE, Holubkov R, Edmundowicz D, et al. Treatment of patients admitted to the hospital with congestive heart failure: specialty related disparities in practice patterns and outcome. J Am Coll Cardiol 1997; 30: 733-8.

18. Bursi F, Weston SA, Redfield MM, Jacobsen SJ, Pakhomov S, Nkomo VT, et al. Systolic and diastolic heart failure in the community. JAMA 2006; 296: 2209-16.

19. Gil M, Maruyat J, Sala J, Masiá R, Elosúa R, Rohlz I y los investigadores del estudio REGICOR. Tendencias en la incidencia y letalidad del infarto agudo de miocardio en Gerona entre 1990 y 1997. Gac Sanit 2000 (Supl. 1): 81. 